\title{
IL MARCHESE DI SANTILLANA E I VOLGARIZZAMENTI ITALIANI DI CICERONE*
}

\author{
Paolo Divizia \\ Masarykova univerzita, Brno \\ paolodivizia@yahoo.it
}

Il ruolo svolto da Íñigo López de Mendoza (1398-1458) - primo marchese di Santillana a partire dal 1445 - come promotore di volgarizzamenti in castigliano (innanzitutto per uso personale) è giustamente messo in evidenza negli studi: di lui si sottolineano il fatto che non conoscesse il latino o non lo conoscesse abbastanza bene da poter leggere i classici in lingua originale, l'approfondita conoscenza della lingua italiana (oltre che del francese, del catalano e del gallego), e l'ampia rete di contatti attraverso cui si procurò una rimarchevole collezione di manoscritti da diversi paesi e in particolare dall'Italia. La biblioteca, collocata nel suo palazzo a Guadalajara, sarebbe poi parzialmente confluita in quella dei duchi di Osuna e dell'Infantado, e quest'ultima verso la fine dell'Ottocento nella Biblioteca Nacional di Madrid'.

\footnotetext{
* Este trabajo se inscribe en el marco del proyecto de investigación Alfonso de Cartagena. Obras Completas FFI 2014-55902-P y FFI 2017-84858-P (MINECO y MICINN, Gobierno de España).

${ }^{1}$ La bibliothèque du marquis de Santillane, par Mario Schiff, Paris, Librairie Émile Bouillon, 1905; Historia de la literatura española [1973], Barcelona, Ariel, 1999 (traduzione di A literary history of Spain, London, Ernest Benn, 1971), vol. I. La Edad Media, al cuidado de Alan D. Deyermond, alle pp. 323325; Peter E. Russell y Francisco Rico, "Caminos del humanismo», in Historia y crítica de la literatura española, dir. da Francisco Rico, Barcelona, Crítica, 1980-1994, vol. I. Edad media, al cuidado de Alan Deyermond [coord. da Modesta Lozano; trad. da Carlos Pujol], pp. 442-449, alle pp. 443-444; Felipe B. Pedraza Jiménez y Milagros Rodríguez Cáceres, «El Marqués de Santillana», in Id., Manual de literatura española, Pamplona, Cénlit Ediciones, 1981-2005, vol. I. Edad Media, pp. 660-681 e 748. In quanto ammiratore e imitatore di Dante, nonché promotore di una traduzione spagnola della Commedia e possessore di due codici del poema oggi conservati a Madrid presso la Biblioteca Nacional de España (ms. 10186; ms. Vitrina 23-2), al marchese è dedicata la voce «Santillana, Íñigo López de Mendoza, marchese di», a cura di Marco Boni, in Enciclopedia Dantesca, Roma, Istituto dell'Enciclopedia Treccani, 1970. Vid. anche «Santillana, Íñigo López de Mendoza, marchese de», a cura di Salvatore Battaglia, in Enciclopedia Treccani, Roma, Istituto dell'Enciclopedia Treccani, 1936 (ma si osservi che la data di nascita del marchese è 1398, non 1389 , data che ebbe invece poi una certa fortuna negli studi di area italiana).
} 
Sulle modalità degli acquisti librari disponiamo di alcuni documenti preziosi. In una lettera non datata al figlio Pedro González (il futuro cardinal Mendoza), quando era studente utrumque iuris a Salamanca (1446-1452), il marchese scrive:

\begin{abstract}
Algunos libros e oraçiones he resçibido por un pariente e amigo mío, este otro día, que nuevamente es venido de Italia, los quales asy por Leonardo de Areçio como por Pedro Caudino [sic] milanés, d'aquel príncipe de los poetas, Homero, e de la historia troyana que él compuso, a la qual Iliade intituló, [fueron] traduçidos del griego a la lengua latina, creo ser primero, segundo, terçero o quarto, e parte del décimo libro. ${ }^{2}$
\end{abstract}

Dal momento che i testi di cui si tratta in questo lavoro risalgono in buona parte al pieno umanesimo, è sorto il dubbio se parlare di volgarizzamenti o traduzioni. La forte polarizzazione tra i due termini individuata e sostenuta da Gianfranco Folena, Volgarizzare e tradurre, Torino, Einaudi, 1991, ha contribuito in ambito scientifico all'affermarsi pressoché totale del primo verbo inizialmente per l'area romanza medievale, e poi - attenuatosi il senso di tale opposizione e prevalendo la percezione di una continuità tra medioevo e umanesimo - fino alla rivoluzione gutenberghiana, come si può vedere ad esempio scorrendo la più recente bibliografia qui sotto citata. In questa sede ci si è dunque adeguati all'uso. Del resto non è sempre facile scegliere tra i due estremi, e il manoscritto oggetto di questo studio - Madrid, Biblioteca Nacional de España, Res. 236 - non offre appigli, in quanto alterna i due termini forse al solo scopo di variatio: il De officiis è «da certo volgarizzato» (c. 1r); il De amicitia, unico testo trecentesco nel manoscritto, è «traducto di latino in volgare» (c. 102r); il libro dei Paradoxa è «da altri volgarizzato» (c. 132r); il De senectute, «da altri volgarizzato» secondo la rubrica (c. 144r), secondo la formula di chiusura «insieme col libro Degli ufficii, Paradoxe è suto tradocto [...] di latino in lingua toscana» (c. 168r). Per un approfondimento della questione vid. Giulio Vaccaro, «Introduzione. Studiare i volgarizzamenti (oggi)?», in Volgarizzamenti: il futuro del passato, a cura di Roman Sosnowski e Giulio Vaccaro, Firenze, Cesati, 2018, pp. 13-33.

Nel riportare le segnature dei manoscritti da qui in avanti si abbrevia secondo il sistema escogitato da Claudio Ciociola, «Indice delle biblioteche citate», in Storia della letteratura italiana, dir. da Enrico Malato, 14 voll., Roma, Salerno Editrice, 1995-2005, vol. X. La tradizione dei testi, coordinato da Claudio Ciociola, 2001, p. LXIII, per cui CV BAV = Città del Vaticano, Biblioteca Apostolica Vaticana; Fi $\mathrm{BNC}=$ Firenze, Biblioteca Nazionale Centrale; Fi BML = Firenze, Biblioteca Medicea Laurenziana; Fi $\mathrm{BR}=$ Firenze, Biblioteca Riccardiana; London $\mathrm{BL}=$ British Library; Madrid BNE $=$ Biblioteca Nacional de España; Paris BNF = Bibliothèque Nationale de France; Ve BNM = Biblioteca Nazionale Marciana. Si citano però in forma completa le biblioteche la cui identificazione potrebbe risultare poco intuitiva in forma abbreviata.

${ }^{2}$ Traggo il testo da La bibliothèque du marquis de Santillane, par Mario Schiff, ob. cit., p. 452, intervenendo lievemente su punteggiatura e diacritici, e integrando «fueron». Schiff propone di identificare il «pariente e amigo» con il poco più giovane umanista Nuño de Guzmán, ma la questione è dibattuta. Pedro-Manuel Cátedra, «Sobre la biblioteca del Marqués de Santillana: La Iliada y Pier Candido Decembrio», in Hispanic Review, 51:1 (Winter 1983), pp. 23-28, a p. 28 n. 17, sottolinea che Nuño de Guzmán comprava libri in buona parte per proprio uso e in parte per altri, inclusi Alfonso de Cartagena e il re Juan II, della cui biblioteca certamente si servì il marchese di Santillana; e osserva che, se l'identificazione è corretta, il marchese nella lettera al figlio farebbe sfoggio di un rapporto privilegiato con Nuño de Guzmán che sarebbe per lo meno da sminuire. Juan Miguel Valero Moreno, «Palabras antiguas para un mundo nuevo: Pier Candido Decembrio, vocabulista, en castellano», in Quaderns d'italià, 22 (2017), pp. 225-240, a p. 232 n. 21, propone più verosimilmente di identificare il (lontano) parente e amico con Íñigo de Ávalos - a cui si allude nella strofa 74 della Comedieta de Ponça del marchese -, al servizio del duca di Milano e in ottimi rapporti sia con Alfonso V (a cui è dedicato un capitolo delle Vite di Vespasiano) sia 
Un'altra testimonianza sugli acquisti di manoscritti effettuati in Italia per conto del marchese di Santillana ci è offerta da Vespasiano da Bisticci nel capitolo delle Vite dedicato al figlio Pedro González de Mendoza:

Faceva continovamente fare libri, et comperava, così sacri come gentili, in modo che ragunò grande quantità di libri, per volere fare una libreria. Aveva il padre signore di primi di quello regno, il quale non era litterato, ma intendeva benissimo la lingua toscana, et per questo fé fare il cardinale qui in Firenze grandissima quantità di libri in lingua toscana, per transferirgli per suo piacere in ispagnuolo, et fece fare in Ispagna in casa sua una libreria di libri toscani, che volle che fussi comune a chi ne voleva, et meser Piero et tutta la sua casa, casa d'uomini nobilissimi, et dati tutti alle virtù, et è oggi in Ispagna de' primi uomini di quello regno ${ }^{3}$.

A prescindere dall'identificazione del «pariente e amigo» citato nella lettera del marchese che abbiamo visto sopra, anche Nuño de Guzmán si rivolse a Vespasiano da Bisticci per rifornirsi di libri. Nel capitolo delle Vite a lui dedicato, «Vita di meser Nugno, di casa reale di Gusmano, casa reale in Ispagna», emerge un ritratto non molto dissimile da quello del marchese, e che fa di lui una delle figure di primo piano nell'emergente umanesimo spagnolo:

Sapeva la lingua toscana maravigliosamente, et legeva in questa lingua meglio che non arebe letto uno toscano, et fece iscrivere infiniti volumi di libri

con umanisti italiani di diversa provenienza. Proprio in virtù della lettera riportata (ove il padre chiede al figlio di volgarizzare il testo dal latino al castigliano) è attribuito a Pedro González de Mendoza il volgarizzamento castigliano anonimo parziale dell'Iliade (London BL Add. 21245, manoscritto appartenuto alla biblioteca del marchese di Santillana) fatto a partire dalle traduzioni latine di Leonardo Bruni e Pier Candido Decembrio.

È da collocare nel medesimo ambiente culturale la trafila di versioni dei Commentarii tres de Primo Bello Punico: scritti in greco da Polibio, tradotti in latino da Leonardo Bruni tra il 1418 e il 1422, volgarizzati in italiano da Pier Candido Decembrio verso il 1438, giunti in Spagna nella biblioteca del marchese di Santillana (ora Madrid BNE 10301), volti in castigliano da un anonimo prima del 1578. Rimando a Jaime González Gómez, «La traducción historiográfica en el Cuatrocientos entre Italia y Castilla. Notas sobre la versión decembriana de la Primera Guerra Púnica y su traducción castellana», in Revista de filología romanica, 34:1 (2017), pp. 103-115. Ringrazio l'autore per aver messo a mia disposizione la sua tesi di dottorato: Id., Las traducciones cuatrocentistas de la Primera guerra púnica de Polibio en italiano y castellano: edición bilingüe y estudio filológico, directora: Lola Pons Rodríguez, Universidad de Sevilla, 2017.

${ }^{3}$ Vespasiano da Bisticci, Le vite, edizione critica con introduzione e commento di Aulo Greco, Firenze, Istituto Nazionale di Studi sul Rinascimento, 1976, vol. I, pp. 205-206 (il capitolo è intitolato semplicemente «Cardinale ispagnuolo»). Mario Schiff (La bibliothèque du marquis de Santillane, ob. cit., pp. LXXXVII-LXXXIX) osserva però che Vespasiano, che scrive vari anni dopo la morte del marchese di Santillana, potrebbe aver confuso il più famoso Pedro con un altro figlio, Íñigo, futuro conte di Tendilla. La testimonianza di Vespasiano rimane comunque di grande rilievo e contribuisce non poco a far luce sul modus operandi del marchese, che è il vero protagonista dell'inizio del capitolo. 
nella lingua toscana, i quali mandò in Spagna [...] [ed era] tanto volto alle lettere toscane, che più volte mandò infino di Spagna qui sua uomini propri, alle sua ispese, a fare trascrivere libri, et istetono in Firenze tanto che furono finiti. Et fece tradurre più libri di latino in lingua tuscana con grande premio, le Tusculane di Tulio, De oratore, le Declamationi di Quintiliano, Macrobio De saturnalibus, de più altre opere, fece in questa lingua una degnissima libraria, le quale, prevento dalla morte in Sibilia, capitorono male 4

Vespasiano non mette in connessione Nuño de Guzmán e il marchese di Santillana, ma dei quattro volgarizzamenti citati, eseguiti «a pititione di messere Nugnio Gusmano spagnuolo», almeno due erano presenti nella biblioteca del marchese: Paris BNF It. 1703 (Cicerone, Tusculanae disputationes) e Madrid BNE Vitr./16/6 (Quintiliano, Declamationes). Entrambi i codici sono esemplari di lusso, confezionati nel 1456 presso la bottega di Vespasiano, e riportano il nome di Nuño de Guzmán ma lasciano bianco lo spazio all'interno del medaglione che avrebbe dovuto accogliere lo stemma del proprietario ${ }^{5}$.

$* * *$

Se è riconosciuto, dicevamo, il ruolo del marchese nel promuovere volgarizzamenti in castigliano, è invece quasi del tutto dimenticata o trascurata la funzione che Íñigo López de Mendoza ebbe nel promuovere volgarizzamenti in lingua italiana. Al di là delle parole di Vespasiano da Bisticci («fé fare [...] libri in lingua toscana») - che comunque paiono riferirsi più propriamente alla copiatura di testi già in toscano che non alla commissione di appositi volgarizzamenti $-{ }^{6}$ e delle illazioni che si potrebbero trarre dai due manoscritti suddetti, delle Tusculanae e delle Declamationes, una notizia certa si ricava da un manoscritto appartenuto alla biblioteca del marchese: Madrid BNE

\footnotetext{
${ }^{4}$ Vespasiano da Bisticci, Le vite, cit., vol. I, pp. 435-441, alle pp. 438 e 440-441.

${ }^{5}$ Luca Bellone, «Le Tusculane di Tulio clarissimo oratore tradocte di latino in volgare fiorentino, a pititione di messere Nugnio Gusmano ispagnuolo», in Carte romanze, 1:1 (2013), pp. 217-242 (consultabile online all'indirizzo <http://riviste.unimi.it/index.php/carteromanze/index $>$ ), pensa che i due volgarizzamenti potessero essere destinati in ultima istanza al marchese, ma vid. quanto detto sopra a proposito del «pariente e amigo». È da ricordare che Nuño de Guzmán dedicò al marchese di Santillana la sua versione in castigliano dell'Orazione a Gismondo Pandolfo de' Malatesta di Giannozzo Manetti, il quale a sua volta aveva scritto per Nuño de Guzmán l’Apologia - come ricorda Vespasiano nel capitolo dedicato a quest'ultimo (Vite, cit., vol. I, pp. 436-437) - permettendogli così di riconciliarsi con il padre. Una riproduzione a colori dei due testimoni è ora disponibile sul sito web delle rispettive biblioteche che li conservano: Bibliothèque nationale de France - Gallica all'indirizzo <http://gallica.bnf.fr> e Biblioteca Nacional de España - Biblioteca Digital Hispanica, all'indirizzo <http://www.bne.es/es/Catalogos/ BibliotecaDigitalHispanica/Inicio/index.html>.

${ }^{6}$ Osservo infatti che Vespasiano, nel capitolo relativo a Nuño de Guzmán, scrive invece in forma esplicita «fece tradurre».
} 
Res. 236, elegante codice membranaceo in scrittura umanistica della metà del Quattrocento, cc. II+168+II' numerate modernamente a lapis (rilegatura moderna, carte di guardia posteriori non originali), 275x190 mm, riconducibile proprio alla bottega di Vespasiano da Bisticci e contenente quattro volgarizzamenti in lingua italiana di Cicerone: De officiis (cc. 1r-101v); Laelius de amicitia (cc. 102r-131v); Paradoxa stoicorum (cc. 132r-143v); Cato maior de senectute (cc. 144r-168r) ${ }^{7}$. La formula di chiusura che segue immediatamente alla fine dell'ultimo trattato a c. 168r, in inchiostro rosso e della stessa mano che ha vergato l'intero codice, è esplicita in proposito: ${ }^{8}$

Finiscie il libro Della senectù composto da Marco Tullio Cicerone, el quale insieme col libro Degli ufficii, Paradoxe, è suto tradocto nella magnifica città di Firenze, di latino in lingua toscana, ad petitione del magnifico et generoso cavaliere messere Nicholops ${ }^{9}$ de Mendoza, marchese di Sanctigliana.

${ }^{7}$ Descrizione del codice disponibile in Mario Schiff, La bibliothèque du marquis de Santillane, ob. cit., pp. 59-60; José Manuel Lucía Megías, «Manuscritos románicos no castellanos (II). Manuscritos en italiano conservados en la Biblioteca Nacional de Madrid (1)», in Revista de Literatura Medieval, 9 (1997), pp. 247-271, alle pp. 262-264; e sul sito web Biblioteca Nacional de España - Biblioteca Digital Hispánica, cit., ove è possibile consultare anche una riproduzione integrale a colori. Sull'impossibilità di ricostruire integralmente la biblioteca del marchese di Santillana vid. Exposición de la biblioteca de los Mendoza del Infantado en el siglo XV, con motivo de la celebración del V centenario de la muerte de don Íñigo López de Mendoza, marqués de Santillana, [al cuidado de Mario Penna], Madrid, Dirección general de archivos y bibliotecas, 1958, in partic. alle pp. 16-17, ove si mette in evidenza che per disposizione testamentaria solo 100 codici della biblioteca del marchese dovettero passare al primogenito Diego Hurtado de Mendoza, e tra questi 100 codici potevano figurare fino a 20 codici che un altro figlio, il già citato Pedro González («mi fijo el Obispo de Calahorra»), doveva ancora inviare.

${ }^{8}$ Questi i criteri di trascrizione, in tutto l'articolo: conservo la grafia, ma divido le parole, introduco maiuscole, punteggiatura e segni diacritici secondo l'uso moderno; sciolgo tra parentesi tonde le abbreviazioni (ma conservo abbreviazioni ancora oggi in uso del tipo M.T. Cicerone), distinguo $u$ e $v$, riduco a $i$ le $j$ conservandole però alla fine dei numeri romani; correggo tra parentesi quadre e in nota indico la lezione del manoscritto oppure, ove pare soluzione più opportuna, segnalo in nota le possibili correzioni o la causa dell'errore. Quando rilevante - ossia in presenza di sviste del copista o per segnalare una mise en page particolare - indico con | il cambio di riga e con || il cambio di pagina.

${ }^{9}$ Il copista evidentemente confonde Íñigo López con Niccolò, come già osservava Mario Schiff, $L a$ bibliothèque du marquis de Santillane, ob. cit., p. 60, che trascrive interamente la formula di chiusura del codice ma senza mettere a frutto le preziose informazioni che si possono ricavare. Un breve accenno alle traduzioni eseguite a petizione del marchese si trova invece in Albinia de la Mare, «New research on humanistic scribes in Florence», in Miniatura fiorentina del Rinascimento, 1440-1525: un primo censimento, a cura di Annarosa Garzelli, Firenze-Scandicci, Giunta regionale toscana-La nuova Italia, 1985, vol. I, pp. 395-600, a p. 429; e in Luciano Formisano, «La cultura italiana nella penisola iberica», in Enrico Malato (direttore), Storia della letteratura italiana, ob. cit., vol. XII. La letteratura italiana fuori d'Italia, coordinato da Luciano Formisano, 2002, sez. I. «Dalle origini all'età dell'Umanesimo», cap. IV. «La diffusione dell'Umanesimo», di Luciano Formisano, Piero Boitani, Vanda Perretta, Francesca Rizzo Nervo, Giacoma Strano, par 6, pp. 141-151 e 187-188 (bibliografia), alle pp. 144-145, ove si citano i quatro volgarizzamenti ciceroniani oggetto del presente lavoro, «che il Marchese aveva fatto acquistare in Italia o direttamente eseguire per lui». 
L'indicazione del titolo nobiliare, conferitogli nel 1445, permette di datare il codice, con tutta probabilità esemplare ufficiale di presentazione, agli anni compresi appunto tra il 1445 e la data della morte avvenuta nel $1458^{10}$.

Nella formula di chiusura si può notare che sono elencati soltanto tre dei quattro volgarizzamenti presenti nel codice. Ma non si tratta di una dimenticanza: come vedremo, il De amicitia, secondo trattato della serie di cui è latore il ms. Res. 236, compare qui secondo un anonimo volgarizzamento trecentesco; per gli altri tre volgarizzamenti siamo invece di fronte a versioni nuove, o parzialmente nuove, che ebbero una circolazione piuttosto limitata e, parrebbe, sempre legata alla produzione libraria della bottega di Vespasiano da Bisticci ${ }^{11}$.

Riprendiamo ora singolarmente le opere tramandate dal manoscritto Res. 236.

\section{De officiis (cc. 1r-101v):}

rubr.: «Incomincia un libro di Marco Tulio Cicerone decto De officiis ad Marco suo figliuolo, da certo volgarizzato»;

inc.: «Advenghadio, o Marco figliuolo, che già uno anno avendo tu udito Cratippo, et questo in Athene, fa bisogno abbondare et di comandamenti et di admaestramenti della philosophia»;

expl.: «se tu tu rallegherai di tali precepti (et) miei ricordi. | Finis».

Edizioni: Volgarizzamento degli Uffici di Cicerone, testo inedito del buon secolo della favella toscana, ora pubblicato da Francesco Palermo, Napoli,

${ }^{10}$ Sul frontespizio, oltre al ritratto di Cicerone nel capolettera, una ricca cornice con motivi vegetali all'interno della quale compaiono animali e putti; su entrambi i lati il motto «Dius e vos» (che in altri mss. provenienti dalla stessa biblioteca, come ad es. Madrid BNE Res. 218, contenente il volgarizzamento italiano vulgato di Valerio Massimo, compare nella forma «Dios e vos»), in basso lo stemma nobiliare del marchese, all'interno di un medaglione sostenuto da cinque putti. Sia il motto sia lo stemma sono legati al conferimento del titolo, ma la loro validità come prova cronologica è inferiore rispetto alla formula di chiusura: lo stemma poteva infatti venire aggiunto in un secondo tempo, come si può vedere ad esempio nei due manoscritti con volgarizzamenti realizzati «a pititione di messere Nugnio Gusmano spagnuolo» di cui si è già detto, nei quali lo spazio all'interno del medaglione destinato ad accogliere uno stemma nobiliare è rimasto bianco. Il medaglione rimasto vuoto si riscontra anche in una nutrita serie di manoscritti riconducibili alla bottega di Vespasiano da Bisticci ora conservati a Città del Vaticano, Biblioteca Apostolica Vaticana, fondo Vaticano Latino, come si può vedere con facilità consultando il sito web della Biblioteca, DVL. Digivatlib, all'indirizzo <https:/digi.vatlib.it/mss/Vat.lat $>$ (ad es. i numeri 174, 189, 192, per citare solo i primi casi, mentre il 259 presenta un'evidente rasura poi non colmata in corrispondenza di un precedente stemma).

${ }^{11}$ Dato che Vespasiano da Bisticci si serviva di numerosi copisti e miniatori senza vincoli di esclusività, in assenza di indicazioni esplicite sui codici, o notizie desumibili dalle Vite o dalle lettere, l'identificazione della bottega va sempre proposta con un minimo di cautela. Espressioni del tipo «Vespasianus librarius Florentinus fecit scribi Florentie»o «fecit fieri»o «transcribendum curavit»o «vendidit» compaiono solo in diciotto codici: vid. Teresa Martínez Manzano, «Un nuevo manuscrito del taller de Vespasiano da Bisticci», in Studi Medievali e Umanistici, 10 (2012), pp. 289-292, alle pp. 290-291. Il problema era già stato segnalato da Albinia de la Mare, «New research on humanistic scribes in Florence», ob. cit., pp. 405-406. 
Tipografia Trani, $1840^{12}$; Volgarizzamento degli Uffici di M. Tullio Cicerone. Testo inedito del buon secolo della lingua in questa seconda edizione napoletana $[. .$.$] recato a miglior lezione da Michele Dello Russo, Napoli, Stamperia$ del Diogene, 1851.

Altri studi: Giuliano Tanturli, «Sulla data e la genesi della Vita civile di Matteo Palmieri», in Rinascimento. Rivista dell'Istituto di studi sul Rinascimento, 36 (1996), pp. 3-48 (alle pp. 22-23 n. 43 segnala altri quattro testimoni del volgarizzamento: Fi BML Plut. 76.75; Fi BNC Magl. xxı.69; Fi BNC Pal. 670; CV BAV Vat. Lat. 7306; e un volgarizzamento diverso conservato in CV BAV Cappon. 51); una scheda sul volgarizzamento risulta in preparazione nel DiVo. Dizionario dei volgarizzamenti, progetto coordinato da Elisa Guadagnini e Giulio Vaccaro, Firenze-Pisa, Opera del Vocabolario Italiano - Scuola Normale Superiore di Pisa, 2012, disponibile online all'indirizzo $<\mathrm{http}$ ://tlion. sns.it/divo/index.php?op=fetch\&type $=$ opera\&lang=it\&id=1099> [ultima consultazione: $12 / 04 / 2018]$.

Nella parte iniziale (De officiis I.1-60) il volgarizzamento tramandato dal manoscritto di Madrid risulta in parte distinto da quello pubblicato da Francesco Palermo, mentre poi il nostro manoscritto e l'edizione a stampa convergono. Un controllo a campione sul ms. CV BAV Urb. Lat. 1142, riconducibile anch'esso alla bottega di Vespasiano da Bisticci, mostra che ove il manoscritto di Madrid e l'edizione Palermo divergono, l'urbinate concorda tendenzialmente con la stampa ${ }^{13}$.

Ma allo stato attuale degli studi sarebbe ancora troppo azzardato stabilire se le innegabili coincidenze tra le due redazioni riscontrabili nella parte iniziale siano dovute a una parentela diretta tra le redazioni stesse o piuttosto alla pedissequa corrispondenza con il modello latino. Non siamo infatti

${ }^{12}$ L'edizione si basa su un non meglio precisato codice riccardiano, da identificare con il codice Fi BR 1602, unico testimone dell'opera presso tale biblioteca descritto in I mss. della R. Biblioteca Riccardiana di Firenze, a cura di Salomone Morpurgo, volume I [e unico], Roma, Presso i principali librai, 1893-1900 («Indici e cataloghi», 15), p. 581. È invece un abbaglio del catalogatore la presenza del $D e$ officiis volgarizzato nel Riccardiano 1213, contenente altre opere di Cicerone (De senectute, Paradoxa, De amicitia) prima secondo il volgarizzamento inedito di frate Filippo predicatore (cc. 17r-81v) e poi in latino (cc. 86r-129r); abbaglio che dipende senza dubbio dalla nota di chiusura (cc. 80r-81v), ove il volgarizzatore, forse da identificare con il copista, dice di aver volgarizzato «delli Officii, de la Vechieçça et de le Paradose», secondo quanto posso dedurre dalla dettagliata scheda sul manoscritto curata da Teresa De Robertis, disponibile sul database MDI. Manoscritti Datati d'Italia, all'indirizzo web $<\mathrm{http}$ ://www. manoscrittidatati.it/> [ultima consultazione: 16/04/2018].

${ }^{13}$ Una nota manoscritta apposta sull'urbinate a c. Ir dice: «Questo volgarizzamento degli Uffici di Cicerone fu pubblicato da Francesco Palermo in Napoli l'an(no) 1840, ma con lezione spesse volte diversa». Si deve però tener conto che si tratta di un'edizione del 1840, in cui lo stesso editore afferma di aver confrontato il volgarizzamento con il testo latino (con tutte le conseguenze immaginabili in un'edizione dell'epoca) e di aver «ripulito e messa in grammatica [...] qualche voce troppo ammuffita e sgrammaticata» (p. III). 
lontani da quanto ha osservato Cristiano Lorenzi a proposito dei volgarizzamenti senechiani, che paiono provenire dallo stesso ambiente culturale:

Nella resa volgare dei tre Dialogi, infatti, è proposta con modalità sempre analoghe una traduzione verbum e verbo, secondo una prassi ben diffusa nel Medioevo. I risultati sono assai modesti: l'autore - privo della necessaria conoscenza del latino e, più in generale, di basilari nozioni di cultura classica - si limita a seguire pedissequamente il dettato del modello, ricalcandone in modo passivo persino il lessico. La traduzione "parola per parola" finisce così per palesare spesso una mancata comprensione del senso del testo originale ${ }^{14}$.

Dovrebbe infine essere stemmaticamente vicino al nostro l'intero codice CV BAV Vat. Lat. 7306, manoscritto miniato che contiene i tre volgarizzamenti “nuovi" del nostro (nell'ordine De officiis, De senectute, Paradoxa) e niente altro" ${ }^{15}$.

\section{Laelius de amicitia, volg. red. B (cc. 102r-131v);} rubr: «Incomincia el libro di Marco Tulio Cicerone Della amicitia da lui composto ad Actico suo amicissimo, traducto di latino in volgare»;

inc.: «Quinto Mutio Augurio Scevola era usato di raccontare molte cose di Gaio Lelio suo suocero»;

expl.: «nessuna altra cosa pensiate o crediate nelle cose essere migliore che lla amistade. | Finis»;

${ }^{14}$ Cristiano Lorenzi, «Un volgarizzamento inedito della Consolatio ad Polybium (ms. Laurenziano Plut. 76.61)», in Bollettino. Opera del Vocabolario Italiano, 17 (2012), pp. 221-243. Mentre per il volgarizzamento delle consolazioni Ad Helviam e Ad Marciam erano noti quattro testimoni (CV BAV Urb. Lat. 1142; CV BAV Ross. 401; Roma, Biblioteca Casanatense, 117; Fi BML Plut. 76.61), per la Consolatio ad Polybium si conosceva il solo laurenziano. Posso ora segnalare un secondo testimone dell'intero terzetto (quinto per le due consolazioni più diffuse): Ve BNM It. VIII 34 (=10386). Oltre all'urbinate - ma non ho visto il rossiano e il casanatense - anche i due testimoni completi sono riconducibili alla bottega di Vespasiano da Bisticci. Il laurenziano contiene soltanto le tre consolazioni senechiane (nell'ordine: $A d$ Marciam, Ad Helviam, Ad Polybium); il marciano reca, oltre alle tre consolazioni senechiane disposte nel medesimo ordine, anche i volgarizzamenti ciceroniani dei Paradoxa e del De senectute (entrambi secondo la stessa versione del ms. Res. 236), e l'apocrifa Oratione d'Escine [sic] agli Ateniesi quando fu confinato. Per il marciano vid. anche la scheda curata da Susy Marcon nel catalogo online Nuova biblioteca manoscritta, Roma, Istituto Centrale per il Catalogo Unico delle Biblioteche Italiane - Ministero dei beni e delle attività culturali e del turismo, 27 luglio 2008, all'indirizzo web $<$ https://manus.iccu.sbn.it// opac_SchedaScheda.php?ID=44219>.

${ }^{15}$ Non ho visto il codice. Sulla presenza di miniature informa Paul Oskar Kristeller, Iter Italicum, London-Leiden, The Warburg Institute-E.J. Brill, 1963-1992, vol. II, 1967, p. 384, che riporta anche gli incipit dei tre volgarizzamenti. Al netto di eventuali contaminazioni, l'identificazione è certa. Per il De senectute, red. $\beta$, vid. Laura Ramello, «Preliminari all'edizione degli antichi volgarizzamenti del 'De senectute'», in Filologia romanza e cultura medievale. Studi in onore di Elio Melli, a cura di Andrea Fassò, Luciano Formisano e Mario Mancini, Alessandria, Edizioni dell'Orso, 1998, pp. 687-713, che sigla il manoscritto RmV2. 
formula di chiusura: «Finiscie il libro composto da Marco Tulio Cicerone, in volgare decto Della amicitia, ad Actico suo amicissimo. Laus Deo».

Edizione della redazione B: Sandro Bertelli, «Il volgarizzamento del De amicitia in un nuovo autografo di Filippo Ceffi (Laurenziano Ashburnham 1084)», in Studi di filologia italiana, 67 (2009), pp. 33-90, edizione del volgarizzamento secondo l'Ashburnhamiano alle pp. 55-90.

Altri studi: Concetto Marchesi, «Le redazioni trecentistiche volgari del De amicitia di Cicerone, secondo i codici fiorentini», in Giornale Storico della Letteratura Italiana, 43 (1904), pp. 312-29; Ovidio, Heroides. Volgarizzamento fiorentino trecentesco di Filippo Ceffi, a cura di Massimo Zaggia, Firenze, SISMEL, 2009, vol. I. Introduzione, testo secondo l'autografo e glossario, p. 121, n. 77 (testimoni red. A) e pp. 121-122, n. 78 (testimoni red. B); Paolo Divizia, «Volgarizzamenti due-trecenteschi da Cicerone e Aristotele in un codice poco noto (Kórnik, Polska Akademia Nauk, Biblioteka Kórnicka, 633)», in Italia Medioevale e Umanistica, 55 (2014), pp. 1-31, alle pp. 2-3 e 18-19 (il ms. di Kórnik è latore della redazione A: a p. 19 «red. B» è dunque da correggere con «red. A»).

Manca ancora uno studio complessivo sui volgarizzamenti del De amicitia, ma fin dall'articolo di Concetto Marchesi è appurato che il volgarizzamento trecentesco è tramandato dai codici in due redazioni, $\mathrm{A}$ e $\mathrm{B}$, di cui la seconda costituisce un rifacimento della prima $^{16}$. Il manoscritto di Madrid conserva il testo della redazione $\mathrm{B}$, come si può con facilità riconoscere tenendo conto del lavoro di Concetto Marchesi e dell'edizione di Sandro Bertelli. Un primo censimento non limitato alle biblioteche fiorentine è stato proposto da Massimo Zaggia, e qualche altro testimone è stato segnalato da chi scrive (vid. i lavori citati sopra). Compulsando l'Iter Italicum, ora nella comoda versione digitale, emerge però che la tradizione è assai più vasta $\mathrm{e}$ che $\mathrm{i}$ volgarizzamenti sono ben più di due: posso citare altri testimoni del volgarizzamento trecentesco (le due redazioni, A e B, presentano lo stesso incipit e perciò non si riescono a distinguere nei cataloghi) - ad es. Roma, Biblioteca Corsiniana, Rossi 408 (44 D 19) e Toledo, Archivo y Biblioteca Capitulares, 10024 -; è invece senza dubbio testimone della redazione B un manoscritto che Kristeller segnalava in una non meglio specificata collezione privata, copiato nel 1458 da Pierozzo di Domenico di Iacopo del Rosso («Pierozzus Dominici Jacobi Rubei»), da identificare con la recente acquisizione Fi BR

\footnotetext{
${ }^{16}$ La redazione $\mathrm{B}$ è la redazione vulgata tra XIV e XV secolo, ma in tempi moderni è più nota la redazione A, perché l'Ottocento purista - pur senza giungere a distinguere nettamente le due redazioni - preferì i manoscritti della prima nell'approntare le stampe del volgarizzamento.
} 
4158 ${ }^{17}$; troviamo poi un volgarizzamento ignoto (veneto?): San Pietroburgo, Biblioteca Nazionale Russa, Razn. Q XVIII 72 (che offre anche altri volgarizzamenti ciceroniani secondo redazioni che a giudicare dall'incipit paiono altrimenti sconosciute: estratti dal secondo libro delle Epistulae familiares e - dopo il De amicitia - il De senectute, i Paradoxa, il Somnium Scipionis e le Tusculanae disputationes $)^{18}$; una manciata di codici della versione di Andrea Cambini che includono sempre anche il De senectute del medesimo volgarizzatore (Fi BNC Magl. XXI 72; CV BAV Chigiano M 4 93; New York, Columbia University Library, Lodge 15); un volgarizzamento forse tardo nel ms. Fi BNC Conv. Soppr. B 3 328; un volgarizzamento francese a opera di Laurent de Premierfait testimoniato da più manoscritti; un volgarizzamento aragonese o castigliano (Madrid BNE 10246, che offre anche il De officiis; e Paris BNF Esp. 533, incompleto). A volte non si capisce di quale versione si tratti, come ad esempio nel ms. London BL Harley 6514.

Infine va segnalata la versione di frate Filippo conservata in Fi BR 1213, già citato sopra a proposito del volgarizzamento del De officiis (non contenuto nel manoscritto).

\section{Paradoxa stoicorum (cc. 132r-143v)}

rubr.: «Incomincia il libro Delle paradoxe, co(m)posto da Marco Tullio Cicerone ad Bruto, da altri volgarizzato»;

inc.: «Spesse volti, o Bruto, io conobbi che quando Catone ad te, zio per madre, orava nel senato, tractava luoghi gravi di philosophia diversi dall'uso publico della corte»;

expl.: «non solamente non copiosi et ricchi sono da essere stimati, ma anchora poveri (et) mendichi. | Finis»;

formula di chiusura: «Qui finisce il libro chiamato Delle paradoxe, composto da Marco Tulio Cicerone ad Bruto».

${ }^{17} \mathrm{Il}$ codice è descritto in I manoscritti datati della Biblioteca Riccardiana di Firenze, IV. Mss. 20014270, a cura di Teresa De Robertis e Rosanna Miriello, Firenze, SISMEL - Edizioni del Galluzzo, 2013, p. 61 e tavola 53 (c. 1r, inizio del De amicitia).

${ }^{18}$ Nell'Iter Italicum, ob. cit., la biblioteca risulta ancora come «Leningrad, Publičnaja biblioteka im. M. E. Saltykova-Ščedrina» (nella versione digitale - Iter Italicum: Accedunt Alia Itinera. On CD-ROM. A Database of Uncatalogued or Incompletely Catalogued Humanistic Manuscripts of the Renaissance in Italian and other Libraries. Compiled by Paul Oskar Kristeller. Consultant Editor Luciano Floridi. Leiden: E.J. Brill, 1995 - mancano i diacritici sulle $\check{c}$ e sulla $\breve{S}$ ). Sul volgarizzamento del Somnium Scipionis tramandato da questo manoscritto vid. quanto scrive Simona Brambilla, «Per la fortuna volgare del Somnium Scipionis: da Zanobi da Strada alla cerchia di Giovanni dalle Celle», in Studi petrarcheschi, XI nuova serie (1994), pp. 200-238, alle pp. 215 e 220. Colgo l'occasione per segnalare una scelta di epistole ciceroniane volgarizzate e abbreviate nel ms. Bologna, Biblioteca Universitaria, it. 357 (361), alle cc. 79r-94r (sul margine superiore di c. 79r si legge della stessa mano «della amicitia», che parrebbe essere il filo conduttore della scelta). Il ms. è cartaceo di fattura fiorentina del terzo quarto del xv secolo. Scrittura mercantesca. Sul frontespizio un medaglione con motivi vegetali sorretto da due angeli. 
Edizioni: Le Paradosse di Marco Tullio Cicerone volgarizzate nel buon secolo di nostra lingua, tratte di un codice Vaticano, di note illustrate e pubblicate dal prof. Giuseppe Spezi scrittore della Vaticana, Roma, Tipografie delle Scienze Matematiche e Fisiche, 1867.

Altri studi: Paradossi, scheda a cura di Cristiano Lorenzi, in DiVo. Dizionario dei volgarizzamenti, ob. cit., disponibile online all'indirizzo <http://tlion.sns. it/divo/index.php?op=fetch\&type $=$ opera\&id $=1093 \&$ lang $=i t>$ [ultima consultazione: 12/04/2018], in cui si segnalano quattro testimoni: CV BAV Rossiano 401; CV BAV Urb. Lat. 1142; CV BAV Vat. Lat. 7306; e il nostro.

Ai manoscritti censiti da Cristiano Lorenzi si deve aggiungere il già citato Ve BNM It. VIII 34 (=10386). Riguardo al codice CV BAV Vat. Lat. 7306 e alla sua vicinanza al nostro, rimando a quanto scritto a proposito del volgarizzamento del De officiis.

Per completezza segnalo che è noto agli studi anche un volgarizzamento dei Paradoxa attribuito a Giovanni delle Celle ${ }^{19}$, mentre pare indipendente dalle due versioni conosciute il volgarizzamento tradito dal manoscritto settecentesco Roma, Biblioteca Vallicelliana, C 76, testimone unico anche di un volgarizzamento del De senectute ${ }^{20}$. Indipendente anche la versione di frate Filippo predicatore del Riccardiano 1213, codice di cui si è già detto sopra.

4. Cato maior de senectute, volg. red. $\beta$ (cc. 144r-168r)

rubr.: «Incomincia il libro chiamato Della senectù, composto da M.T. Cicerone ad Actico, da altri volgarizzato»;

inc.: «Se io alquanto, o Tito, t'aiuto et lievoti la molestia che al presente ti tormenta»;

expl.: «acciò che voi possiate lodare, provati in facti, quelle cose le quali voi havete udito da me. | Finis»;

formula di chiusura: «Finiscie il libro Della senectù composto da Marco Tullio Cicerone, el quale insieme col libro Degli ufficii, Paradoxe, è suto tradocto nella magnifica città di Firenze, di latino in lingua toscana, ad petitione del magnifico et generoso cavaliere messere Nicholops de Mendoza, marchese di Sanctigliana».

${ }^{19}$ Giovanni dalle Celle (attr.), Paradossi, scheda a cura di Cristiano Lorenzi, in DiVo. Dizionario dei volgarizzamenti, cit., disponibile online all'indirizzo <http://tlion.sns.it/divo/index.php?op= fetch\&type=opera\&id=1032\&lang=it $>$ [ultima consultazione: $12 / 04 / 2018]$.

${ }^{20}$ Scheda di Valentina D'Urso nel catalogo online Nuova biblioteca manoscritta, cit., 24 luglio 2008, all'indirizzo <https://manus.iccu.sbn.it//opac_SchedaScheda.php?ID=16326>; per il De senectute vid. Laura Ramello, «Preliminari all'edizione degli antichi volgarizzamenti del 'De senectute'», cit., che sigla il vallicelliano $\mathrm{RmVc}$. 
Edizioni della redazione $\beta$ : non disponibili.

Altri studi: Laura Ramello, «Preliminari all'edizione degli antichi volgarizzamenti del De senectute», art. cit.

Nel censimento proposto da Laura Ramello, che individua sei redazioni, er il volgarizzamento $\beta$ sono segnalati il nostro codice (siglato $\mathrm{MdN}$ ) e il già citato CV BAV Vat. Lat. 7306 (siglato RmV2). A questi due testimoni si devono aggiungere almeno CV BAV Urb. Lat. 1142; e Ve BNM It. VIII 34 (=10386): entrambi riconducibili, come il madrileno, alla bottega di Vespasiano da Bisticci ${ }^{21}$, e piuttosto vicini per contenuto, come si vedrà meglio più avanti.

Posso aggiungere un testimone della redazione $\alpha$ : Madrid BNE 10227. A tale redazione andranno assegnati anche CV BAV Barb. Lat. 4119, segnalato dalla Ramello come «Roma, Bibl. Barberina, 2233» con l'avvertimento che si tratta di una vecchia segnatura; e Milano, Biblioteca Braidense, Morbio 7, elencato ma non esaminato ${ }^{22}$.

Costituisce invece una settima redazione a sé la versione conservata nel codice CV BAV Urb. Lat. 1149, databile alla fine del XVI secolo o agli inizi del XVII ${ }^{23}$.

Da quanto osservato, emerge che la circolazione dei nuovi volgarizzamenti fu minima ${ }^{24}$, e legata a una clientela ricca che si riforniva presso Vespasiano da Bisticci o comunque nel suo ambiente. L'acquisto da parte di committenti ricchi, e a volte stranieri, che spesso facevano tradurre per sé opere latine, non favorì la circolazione dei testi: ma in Spagna fu all'origine di nuove traduzioni, dall'italiano al castigliano (o in altri casi dall'italiano al catalano) ${ }^{25}$. A corollario di ciò, ci si può chiedere chi fossero i volgarizzatori delle opere latine. I codici tacciono il nome del volgarizzatore (l'autore latino e il nome del

${ }^{21}$ Sul vaticano latino non mi pronuncio, non avendolo ancora potuto vedere. Ma l'ambiente in cui fu prodotto è senza dubbio lo stesso.

${ }^{22}$ Laura Ramello, «Preliminari all'edizione degli antichi volgarizzamenti del De senectute», art. cit., p. 690 n. 13 e p. 691.

${ }^{23}$ Riproduzione digitale a colori disponibili sul sito web della Biblioteca, DVL. Digivatlib, ob. cit.

${ }^{24}$ La vasta diffusione dei volgarizzamenti del De senectute di cui si è parlato è dovuta alla presenza di più redazioni, e al successo in particolare del volgarizzamento $\alpha$; situazione analoga per i volgarizzamenti del De amicitia, con la fortuna del volgarizzamento trecentesco (red. A e soprattutto B).

${ }^{25}$ Dovrebbe invece constituire un episodio a sé la versione castigliana cinquecentesca del De amicitia e del De Senectute a opera di Cristóbal de Castillejo, umanista legato alla corte di Vienna, tramandata dal solo Wien, Österreichische Nationalbibliothek, cod. 12817. Non ho potuto vedere l'edizione disponibile in Cristóbal de Castillejo, Obra completa, edición e introducción de Rogelio Reyes Cano, Madrid, Fundación José Antonio de Castro, 1998, pp. 785-865. 
committente sono in tale contesto prominenti), ma offrono la possibilità, assieme alle Vite di Vespasiano da Bisticci e alla sua corrispondenza epistolare, di fare qualche ipotesi: la frequenza con cui questi volgarizzamenti si trovano in codici riconducibili alla bottega di Vespasiano, il ricorrere di espressioni come «fece tradurre» nelle Vite - celebrazione di intellettuali contemporanei, ma anche manifestazione dell'orgoglio del libraio che ha soddisfatto i loro bisogni culturali e di gloria -, le continue richieste di traduzioni che si leggono nelle lettere a Vespasiano giunte fino a noi ${ }^{26}$, sono tutti elementi che ci portano nella stessa direzione. Vespasiano - oltre a occuparsi della ricerca degli esemplari da copiare o dei libri usati da rimettere sul mercato, e degli aspetti più materiali della produzione libraria (copiatura, miniatura, rilegatura) che affidava a una nutrita schiera di collaboratori - doveva disporre anche di volgarizzatori che lavoravano su richiesta e in anonimato, a maggior gloria del committente. L'edizione organica dei testi e uno studio traduttologico saprà dirci di più su queste figure, ma basti per ora aver indicato la via.

Come esempio concreto della stretta parentela, di produzione e strutturale, e dunque testuale, che si può individuare tra i latori dei volgarizzamenti suddetti riporto in sinossi la tavola (verso contrapposto al frontespizio) di tre codici riconducibili a Vespasiano citati più volte in questo lavoro:

\begin{tabular}{|c|c|c|}
\hline MADRID BNE RES. 236 & CV BAV URB. LAT. 1142 & VE BNM Iт. VIII 34 (=10386) \\
\hline $\begin{array}{l}\text { In questo libro si contengono } \\
\text { le infrascripte opere, cioè: }\end{array}$ & $\begin{array}{l}\text { In questo volume si con- } \\
\text { tengono le infrascripte } \\
\text { opere, cioè: }\end{array}$ & $\begin{array}{l}\text { In questo volume si conten- } \\
\text { gono le infrascripte chose no- } \\
\text { minate da piè: }\end{array}$ \\
\hline \multirow{5}{*}{$\begin{array}{l}\text { Tullio de offitiis libri .iij.; } \\
\text { Tullio de amicitia libro .j.; } \\
\text { Tullio de paradoxis libro .j.; } \\
\text { Tullio de senectute libro.j. }\end{array}$} & $\begin{array}{r}\text { Tre }^{\text {a libri degli officii }} \\
\text { di Tullio Cicerone ad }\end{array}$ & $\begin{array}{l}\text { Una consolatione di Senecha } \\
\text { a Martia; }\end{array}$ \\
\hline & Marco suo figluolo; & Una consolatione ad Elbia \\
\hline & $\begin{array}{l}\text { ad Martia nella morte } \\
\text { del figluolo; }\end{array}$ & $\begin{array}{l}\text { Una consolatione del decto a } \\
\text { Polibo suo amico; }\end{array}$ \\
\hline & $\begin{array}{l}\text { Consolatione di Seneca } \\
\text { ad Elbia sua madre; }\end{array}$ & $\begin{array}{l}\text { Tullio de senectù; } \\
\text { Paradosse di Tullio; }\end{array}$ \\
\hline & $\begin{array}{l}\text { Paradoxe di M. Tullio ad } \\
\text { Bruto; } \\
\text { Libro della senectù di M. } \\
\text { Tullio Cicerone. }\end{array}$ & $\begin{array}{l}\text { Orazione d'Escine agl'athe- } \\
\text { niesi tradotte di greco in } \\
\text { latino e di latino in volgare; }\end{array}$ \\
\hline
\end{tabular}

${ }^{\mathrm{a}}$ Tre] scritto su rasura, con tutta probabilità su un precedente $Q$ [uattro].

\footnotetext{
${ }^{26}$ Vespasiano da Bisticci, Lettere. A digital edition, responsabile dell'edizione digitale: Francesca Tomasi, Università di Bologna, 2013, consultabile all'indirizzo: <http://vespasianodabisticciletters.unibo.it/> . Purtroppo non sono conservate lettere dei committenti spagnoli di cui si parla nel presente lavoro.
} 
Anche il Vat. Lat. 7306 andrebbe collocato in questo schema, ma purtroppo finora non ho avuto accesso al codice. I dati da raccogliere sono ancora molti, ma mi pare che stia emergendo un ambiente culturale molto fervido che soltanto un approccio multidisciplinare, improntato a un'idea di filologia allargata, potrà illuminare pienamente ${ }^{27}$.

Un discorso a parte meriterebbe il codice Madrid BNE $10227^{28}$, che contiene il volgarizzamento del De senectute secondo la redazione $\alpha^{29}$. Di origine fiorentina, e appartenuto inizialmente a un fiorentino - sull'ultima carta, c. 95r, si legge: "Questo libro è del nobile huomo Teri di Lorenço di Teri, hono(revol)e cittadino fiorentino, il quale gli scripsi io Ciaio di Pagolo di Ciaio, cittadino (e) not(aio) fiorentino» -, il codice giunse in Spagna, o nelle mani di uno spagnolo, già nel XV secolo, come testimoniano alcune note in castigliano sui margini, o più spesso in interlineo, nelle primissime carte del $D e$ senectute: note che si limitano a tradurre qualche parola o espressione (ad es. in corrispondenza di giova: ayuda; malagevole: grave; stultitia: locura) o a segnalare parole sdrucciole (essere: acento breve) o lo scioglimento di qualche abbreviazione, come nel caso di quella per cum/con/com: «esta let(ra) ' 9 ' es ta(n)to $\operatorname{com}(m) \mathrm{o}^{30}$ 'co(n)'».

Il codice, pergamenaceo in scrittura umanistica, presenta una decorazione (aggiunta in un secondo tempo?) limitata ai capilettera, ma di qualche pregio, con bianchi girari e bordi in azzurro, e foglia d'oro. Sul margine inferiore di c. 1r (su rasura?) lo stemma nobiliare in foglia d'oro dei Figueroa, famiglia da cui proveniva la prima moglie del marchese di Santillana, Catalina Suárez de Figueroa: va però detto che questo non fu l'unico matrimonio tra le due famiglie. Appartenne alla biblioteca duchi di Osuna e dell'Infantado, ma non ci sono elementi certi per ritenere che il codice sia appartenuto al marchese ${ }^{31}$.

${ }^{27}$ Un aiuto inimmaginabile fino a pochi anni fa viene senza dubbio dalle tecnologie digitali, e dalla lodevole prassi di alcune biblioteche (come la Biblioteca Nacional di Madrid, la Biblioteca Vaticana, la Biblioteca Medicea Laurenziana e la Bibliothèque nationale di Parigi) di mettere a disposizione online almeno una parte delle loro ricche collezioni manoscritte, permettendo così allo studioso (oltre che un notevole risparmio di tempo e denaro) di affiancare sul proprio tavolo, o meglio sul proprio schermo, codici che altrimenti non si potrebbero compulsare in contemporanea.

${ }^{28}$ Descrizione e riproduzione a colori disponibile sul sito web BNE - Biblioteca Digital Hispánica, ob. cit.

${ }^{29} \mathrm{Il}$ testimone, si è già detto, non è contemplato nel censimento fornito da Laura Ramello, «Preliminari all'edizione degli antichi volgarizzamenti del De senectute», art. cit.

${ }^{30}$ Titulus sovrabbondante.

${ }^{31}$ Dello stesso parere già Mario Schiff, La bibliothèque du marquis de Santillane, ob. cit., pp. 329-331, ove il manoscritto non è contrassegnato dall'asterisco posto «en tête de toutes les notices consacrées à des 
Mi limito qui a osservare che il contenuto del codice - Trattatello in laude di Dante di Boccaccio, le quindici canzoni 'distese' di Dante ${ }^{32}$, quattro orazioni di Stefano Porcari ${ }^{33}$, il volgarizzamento del De senectute - lo avvicina al manoscritto Fi BR 1083, composto di due unità codicologiche riunite già anticamente, la prima delle quali (cc. 1-71) offre nello stesso ordine le stesse opere contenute nel codice Madrid BNE 1022734; e al manoscritto London BL Harley 4082, in cui però mancano le quindici canzoni distese di Dante, e il Trattatello in laude di Dante è collocato in fondo ${ }^{35}$. Mi occuperò in altra sede del codice e in particolare del volgarizzamento del De senectute, assieme ad altri testimoni individuati di recente.

Postilla [novembre 2018]: A lavoro terminato ho potuto consultare il codice CV BAV Vat. Lat. 7306. Confermo quanto deducevo per via indiziaria, ossia che anche tale manoscritto presenta le stesse caratteristiche codicologiche degli altri tre codici strettamente imparentati, e riporto qui quanto si legge sul controfrontespizio (c. IVv), entro medaglione, in lettere maiuscole: «In questo volume si contengono libri .iii. Degli ufitii di Tulio, e Tulio Della vechiaia et le Paradoxe».

Recibido: $31 / 05 / 2018$

Aceptado: 26/07/2018

manuscrits dont l'étude nous a permis d'affirmer qu'ils ont appartenu au marquis de Santillane» (p. xx); Mario Penna, Exposicion de la biblioteca de los Mendoza del Infantado en el siglo XV, ob. cit., p. 33, num. 28 (che adotta lo stesso sistema degli asterischi); e la descrizione disponibile sul sito web $B N E$ - Biblioteca Digital Hispánica, ob. cit. Ė invece assegnato alla biblioteca del marchese da José Manuel Lucía Megías, «Manuscritos románicos no castellanos (II). Manuscritos en italiano conservados en la Biblioteca Nacional de Madrid (2)», in Revista de Literatura Medieval, 10 (1998), pp. 185-206, alle pp. 199-202.

${ }^{32} \mathrm{Su}$ cui vid. da ultimo Marco Grimaldi, «Boccaccio editore delle canzoni di Dante», in Boccaccio editore e interprete di Dante, Atti del convegno internazionale di Roma (28-30 ottobre 2013), a cura di Luca Azzetta e Andrea Mazzucchi, Roma, Salerno Editrice, 2014, pp. 137-157, con ampia bibliografia.

${ }^{33}$ Questi gli incipit: «Quante volte io riguardo»; «Io mi ricordo»; «Se alcuna volta è stato smarrito»; «Molte considerationi m'occorrono». Corrispondono alle prime quattro orazioni pubblicate in Prose del giovane Buonaccorso da Montemagno, inedite alcune, da due codici della Biblioteca Capitolare di Vero$n a$, per mons. G. B. C. Giuliari, Bologna, Gaetano Romagnoli, 1874 («Scelta di curiosità letterarie», 141). All'epoca Stefano Porcari non ne era ancora stato identificato come autore.

${ }^{34}$ Tra i 109 testimoni delle orazioni di Stefano Porcari censiti da Massimo Miglio, «"Viva la libertà et populo de Roma". Oratoria e politica: Stefano Porcari», in Palaeographica diplomatica et archivistica. Studi in onore di Giulio Battelli, a cura della Scuola speciale per archivisti e bibliotecari dell'Università di Roma, I, Roma, Edizioni di storia e letteratura, 1979, pp. 381-428, il codice Fi BR 1083 (siglato 50) è l'unico che presenti solo le prime quattro orazioni nell'ordine I II III IV - ma va detto che il censimento si limita all'Italia e alla Biblioteca Vaticana -. Per una descrizione dettagliata del riccardiano vid. I mss. della R. Biblioteca Riccardiana di Firenze, a cura di Salomone Morpurgo, ob. cit., pp. 78-79, da cui ricavo l'informazione sulle due unità codicologiche.

${ }^{35}$ Descrizione in Iter Italicum: Accedunt Alia Itinera. On CD-ROM, ob. cit. 


\title{
$\cos$
}

\author{
IL MARCHESE Di SANTILlana \\ E I VOLGARIZZAMENTI ITALIANI DI CICERONE
}

RESUMEN: El presente trabajo es un estudio del manuscrito Res. 236 de la Biblioteca Nacional de España, que contiene una serie de traducciones al florentino de Cicerón (De officiis, Paradoxa y De senectute) encargadas por el Marqués de Santillana, además de una traducción del De amicitia del siglo xIv. A partir de los datos aportados por este manuscrito y por otras fuentes, además del papel desempeñado por el Marqués en el encargo de traducciones al florentino, se investiga: (a) su circulación limitada, (b) la actividad del taller del librero Vespasiano da Bisticci y (c) el hecho de que este último tuviera un grupo de traductores a disposición para satisfacer las solicitudes de su clientela más adinerada.

Palabras clave: Marqués de Santillana. Cicerón. Traducciones. Florentino. Manuscritos.

\section{The Marquis of Santillana \\ AND THE ITALIAN VERNACULAR TRANSLATIONS OF CICERO}

\begin{abstract}
This article provides a study of the manuscript Res. 236 of the National Library of Spain, which contains a series of Florentine vernacular translations from Cicero (De officiis, Paradoxa and De senectute) sponsored by the Marquis of Santillana and a thirteenth century translation of the De amicitia. Based on the data provided by this manuscript and other sources, in addition to the role played by the Marquis in the sponsorship of translations into Florentine vernacular, (a) their very limited circulation, (b) the activity of book dealer Vespasiano da Bisticci workshop, and (c) the fact that the latter had a group of translators at disposition to satisfy the requests of his well-off clientele are investigated.
\end{abstract}

KeYwords: Marquis of Santillana. Cicero. Vernacular translations. Florentine. Manuscripts. 